\title{
Magnetic monopole mechanism for localized electron pairing in HTS
}

\section{Cristina Diamantini}

University of Perugia

\section{Carlo Trugenberger}

SwissScientific Technologies SA

Valerii Vinokur ( $\square$ vmvinokour@gmail.com )

Terra Quantum AG https://orcid.org/0000-0002-0977-3515

\section{Article}

Keywords: Effective Field Theory, Pseudogap Phase, Charge Condensate, Condensate of Dyons, Superconductor-insulator Transition, Josephson Links

Posted Date: July 15th, 2021

DOI: https://doi.org/10.21203/rs.3.rs-694994/v1

License: (c) (i) This work is licensed under a Creative Commons Attribution 4.0 International License. Read Full License 


\title{
Magnetic monopole mechanism for localized electron pairing in HTS
}

\author{
M. C. Diamantini, ${ }^{1}$ C. A. Trugenberger, ${ }^{2}$ \& V. M. Vinokur ${ }^{3, *}$
}

Recent effective field theory of high-temperature superconductivity (HTS) captures the universal features of HTS and the pseudogap phase and explains the underlying physics as a coexistence of a charge condensate with a condensate of dyons, particles carrying both magnetic and electric charges. Central to this picture are magnetic monopoles emerging in the proximity of the topological quantum superconductor-insulator transition (SIT) that dominates the HTS phase diagram. However, the mechanism responsible for spatially localized electron pairing, characteristic of HTS remains elusive. Here we show that real-space, localized electron pairing is mediated by magnetic monopoles and occurs well above the superconducting transition temperature $T_{\mathrm{c}}$. Localized electron pairing promotes the formation of superconducting granules connected by Josephson links. Global superconductivity sets in when these granules form an infinite cluster at $T_{\mathrm{c}}$ which is estimated to fall in the range from hundred to thousand Kelvins. Our findings pave the way to tailoring materials with elevated superconducting transition temperatures.

Despite more than three decades of tireless efforts, the nature of high-temperature superconductivity (HTS) have been remaining a mystery ${ }^{1-3}$. An effective field theory ${ }^{4}$ resolved it and proposed that the universal features of HTS and the equally mysterious pseudogap phase follow from the coexistence of a charge condensate with a condensate of dyons, particles carrying both magnetic and electric charges. The major role in the HTS mechanism is taken by magnetic monopoles emerging in the vicinity of the topological quantum superconductor-insulator transition (SIT) that dominates the HTS phase diagram. The remaining piece of the HTS puzzle is the pairing mechanism which has to differ from that in conventional superconductors: in HTS the electron pairing should be spatially localized and result in the pair size comparable or less than the distance between the pairs ${ }^{5}$. Here we show that real-space, localized electron pairing is mediated by magnetic monopoles and occurs well above the superconducting transition temperature $T_{\mathrm{c}}$. Localized electron pairing promotes the formation of superconducting granules connected by Josephson links, and global superconductivity sets in when these granules form an infinite cluster at $T_{\mathrm{c}}$.

The discovery of HTS about 40 years ago promised a new era of industrial and technological advances, with power-loss free electric grid lines spanning continents and superconducting kitchen appliances in every household. Yet, materials superconducting at room temperature are still a dream. The lack in understanding of the HTS pairing ${ }^{1-3}$ hindered the desired progress. So far, neither Anderson's resonating valence bond (RVB) theory of HTS based on electron correlations ${ }^{6}$ and its recent development $^{7}$, nor the alternative route, deriving HTS from Cooper pairing near a quantum critical point (QCP) associated with an antiferromagnetic order-itinerant electron spin transition ${ }^{8,9}$, while beautifully capturing many important features ${ }^{1,2,10-22}$ of the superconducting and the related pseudogap phase, succeeded to provide a general, unifying theory of HTS.

From the relevance of quantum criticality for $\mathrm{HTS}^{9}$ one can derive the superconducting transition temperature as $T_{\mathrm{c}} \sim E_{\mathrm{F}} / \sqrt{g}$, with $E_{\mathrm{F}}$ being the Fermi energy and $g$ being the coupling constant, in remarkable contrast to the standard BCS behavior, $T_{\mathrm{c}} \sim \exp (-$ const $/ g)$. Yet, a strong coupling has to be assumed, and the characteristic feature of HTS, a small superconducting coherence length $\xi$, implying non-overlapping Cooper pairs, is not explained. It has been suggested that the path towards a better understanding of the emergence of superconductivity lies in investigating its breakdown via the superconductor-insulator transition (SIT) ${ }^{23}$ and the emergent phases around it ${ }^{24,25}$. However, while the relevance of the SIT is mostly accepted, there are still many aspects that do not fit the observed HTS phenomenology, mostly originating from the fact that the SIT is a phenomenon well understood only in 2D.

A field-theoretical approach establishing the long sought unified theory of HTS and accounting for all universal features within the superconducting dome and in the pseudogap state in a consistent whole ${ }^{4}$, stemmed from the topological gauge theory of the SIT $^{24,26}$ and its generalization to three dimensions ${ }^{27}$. A fundamental mechanism of the HTS was identified as the formation of a condensate of dyons, electrically charged magnetic monopoles ${ }^{4}$. The superconducting state is a coexistence phase of dyon and pure charge condensates. The pseudogap state above $T_{\mathrm{c}}$ is a new state of matter, the oblique superinsulator, which harbors a pure dyon condensate and exhibits magnetoelectric and nematic effects. Symmetry-protected fermionic surface dyons of charge $2 e$, living on a percolation network, cause an electric resistance proportional to the square of the temperature, in a full accord with the experiment ${ }^{1}$. The elevated $T_{\mathrm{c}}$ results from the topological obstruction of splitting Cooper pairs into single electrons by magnetic monopoles.

Crucial in this picture is the emergent self-induced granularity ${ }^{4,27}$ characterizing the SIT at the antiferromagnetic quantum critical point (AFM-QCP) ${ }^{12,20}$. Superconducting granules of the spatial scale of order of a few superconducting coherence lengths $\xi$ and connected by tunnelling links produce a medium supporting magnetic monopoles ${ }^{27-29}$ and dyons ${ }^{4}$. The last missing piece to complete this picture of HTS is a mechanism of localized electron pairing in real space.

Here we demonstrate that this long-sought HTS pairing mechanism is the binding of electrons by magnetic monopoles. The minimal model of a HTS material is taken as two conducting planes separated by a distance $s$ of the atomic scale. Magnetic monopoles emerging between the planes bind two electrons, residing on the respective opposite planes, into a state of higher angular momentum. The radical difference of the monopole-based binding mechanism from other commonly considered mechanisms ${ }^{7,9,22,30}$ is that electron pairs are spatially localized around the monopoles. The heavy monopoles anchoring the electron pairs serve, thus, as nucleation points for the granular structure that emerges upon cooling the system from the temperature of pair formation, $T_{\text {pair }}$ down to $T_{\mathrm{c}}$. In a system supporting a sufficient monopole density, global superconductivity sets in when the droplets comprising the electron pairs and linked by tunnelling junctions form an infinite cluster. It thus occurs as the temperature compares with the coupling energy, and our estimate gives $T_{\mathrm{c}}=O\left(10^{2}\right) \mathrm{K}$ for a typical granule size of $O(1) \mathrm{nm}$. We stress that these purely magnetic monopoles are different from the dyons in the pseudogap condensate ${ }^{4}$. The former are fluctuation-induced topological 


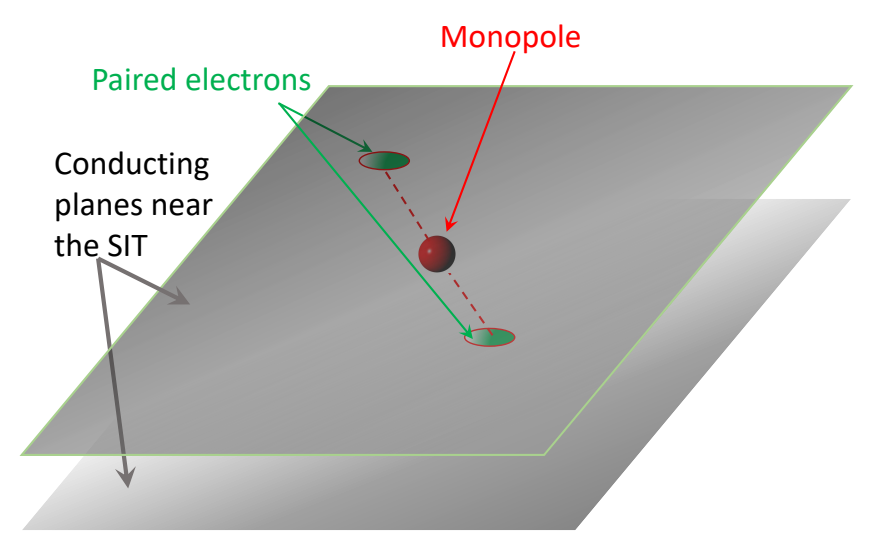

Figure 1 | A sketch of the minimal model for HTS. The conducting planes near the SIT are shown in gray. Heavy monopole appears in the middle between the planes and paired electrons are located in the opposite planes, their motion being restricted to respective planes.

excitations which become stable since electron pairing reduces their repulsive energy and hence the total energy of the system. The pseudogapresiding monopoles are the endpoints of Josephson-like vortices residing in the granular array.

\section{Electron pairing by a single monopole}

We start our derivation by considering two electrons interacting via the spherically symmetric repulsive $1 / r$ Coulomb potential (we use natural units $c=1, \hbar=1, \varepsilon_{0}=1$ ) and the short-range repulsion potential $V_{\mathrm{R}}(r)$. Decomposing the wave function into spherical harmonics yields an additional $\ell(\ell+1) / r^{2}$ repulsive centrifugal barrier for states with the angular momentum $\ell>0$. If the short-range repulsion $V_{\mathrm{R}}(r)$ is stronger than the centrifugal barrier, the electrons settle into a state with the sufficiently high angular momentum. Due to rotational symmetry, the $z$ component $m,|m| \leq \ell$, of the angular momentum falls out of the Hamiltonian. What happens, however, if a spherical-symmetry-breaking mechanism encoded in a vector potential makes the Hamiltonian explicitly dependent on the $z$-component of angular momentum? On a dimensional ground, this brings an additional $1 / r^{2}$ term that can have either negative or positive sign. If negative, it can counterbalance the centrifugal barrier resulting in the attractive $1 / r^{2}$ potential at intermediate distances before the Coulomb repulsion takes over. Then a potential well forms, resulting in a discrete spectrum of bound states with finite angular momentum.

Magnetic monopoles provide precisely such a spherical-symmetrybreaking mechanism ${ }^{31}$. In presence of a magnetic monopole of strength $g$ an electron acquires an additional angular momentum $L_{\mathrm{M}}=(e g / 4 \pi) \hat{\mathbf{r}}$, with $\hat{r}$ being the unit vector pointing from the monopole to the electron. The Dirac quantization, $e g=2 \pi n, n \in \mathbb{Z}$, requires that this additional angular momentum contribution, originating from the interplay of the electric and magnetic fields of two point particles matches the spectrum imposed by the rotation group. This is just a spherical symmetry breaking contribution to the angular momentum since it singles out the vector connecting the monopole and electron. As we now show, this monopoleinduced angular momentum can bind electrons, and the optimal angular momentum of the resulting pair depends on the monopole density. The lower densities favor higher angular momenta and vice versa.

Importantly, monopoles change the statistics of original electrons, which become bosons themselves for $e g / 2 \pi$ odd but remain fermions for the even values of $e g / 2 \pi,{ }^{32}$. The centrifugal barrier cancellation takes place only for even values of $e g / 2 \pi$ leaving the original fermionic statistics of the electrons unchanged. Magnetic monopoles, thus induce higher-angular-momentum pairing of electrons. Paired electrons can then Bose condense into droplets localized near the monopole. Because of the Dirac quantization, magnetic monopoles are heavy excitations, with the mass $m_{\mathrm{M}} \propto 1 / \alpha$, with $\alpha=e^{2} / \hbar c \approx 1 / 137$ being the fine structure constant. The droplets are anchored in space and mediate a pairing mechanism giving rise to high- $T_{\mathrm{c}}$ superconductivity. Since the product of the electric and the magnetic charge is $\mathrm{O}(1)$ due to the Dirac quantization condition, this is automatically a strong-coupling pairing mechanism, without any further assumption.

Note the dimensional dichotomy of the HTS materials. From the viewpoint of the charge- and magneto-transport, HTS materials exhibit a profoundly $2 \mathrm{D}$ behavior ${ }^{33,34}$. At the same time phenomena related to topological aspects of the electronic spectrum, like the magnetoelectric effect in the pseudogap state ${ }^{35}$, require the full underlying 3D microscopic nature. The proposed pairing mechanism is aligned with this dichotomy. The monopole pairing rests on a 3D quantum mechanical structure comprising two conducting planes separated by an atomic scale distance so that the charges can tunnel between the planes, providing robust Josephson links between them. At the same time, as long as the thermal coherence length $L_{\mathrm{T}}=\sqrt{2 \pi D /\left(k_{\mathrm{B}} T\right)}$ exceeds the interplane distance $s$, the system exhibits two-dimensional transport properties. Here $D=(\pi / 2 \gamma)\left(k_{\mathrm{B}} T_{\mathrm{c}} / e B_{\mathrm{c} 2}(0)\right)$ is the diffusion length and $\gamma=1.781$ is Euler's constant. Since the dephasing length $L_{\phi}=\sqrt{D \tau_{\phi}} \gg L_{\mathrm{T}}$ as long as $k_{\mathrm{B}} T \gg / \tau_{\phi}$, the quasiparticle description holds well in this $2 \mathrm{D}$ electric response regime and the $3 \mathrm{D}$ quantum mechanical consideration of electron binding applies. This explains why a cuprate monolayer consisting of two conducting planes retains the same high transition temperature ${ }^{36}$ as a $3 \mathrm{D}$ sample, whereas $2 \mathrm{D}$ films of conventional superconductors have $T_{\mathrm{c}}$ much lower than the bulk of the same material.

Let us consider a heavy magnetic monopole of charge $g$ formed in the middle between the two conducting planes. Next, using the remarkable result of ${ }^{37}$ that the quasiparticle lifetime within the layer is proportional to the intraplane scattering rate, we conclude that electrons are bound to intraplane motion with rare interplane hops, and that this intraplane electron motion becomes even more pronounced with increasing disorder and doping. We obtain a three-body quantum mechanical problem involving two electrons of charge $e$ restricted to respective parallel planes and with short-range repulsion, and a Dirac magnetic monopole of magnetic charge $g$ in between. We proceed with the simpler formulation of an infinitely heavy magnetic monopole located at the center of mass of the two electron system. This reduces to the one-body problem of an electron of reduced mass $m / 2$ in the external field of the magnetic monopole, which is amenable to an analytical, albeit approximate solution, yet maintaining the generality of the monopole pairing mechanism.

Dirac monopoles are particles carrying an attached Dirac string. If the string is aligned with the negative $z$-axis, the vector potential $\mathbf{A}_{u}$ of the monopole is ${ }^{31}$

$$
\begin{array}{r}
\mathbf{A}_{u}=f_{u}(r, \theta) \hat{\varphi}, \\
f_{u}(r, \theta)=\frac{g}{4 \pi r} \frac{1-\cos (\theta)}{\sin (\theta)},
\end{array}
$$

which has a singularity at $\theta=\pi$. Here $r, \theta$ and $\varphi$ denote the usual spherical coordinates and $\hat{\varphi}$ is the unit vector in the $\varphi$ direction. To solve the eigenstate problem in the field of a Dirac monopole one cannot use a single set of coordinates for the whole sphere but must use the $\mathrm{Wu}$ Yang formalism ${ }^{38}$ to cover the sphere with a so-called atlas of maps, supplemented by gauge transformation conditions on the overlap regions between the different maps. The simplest atlas comprises two maps, the northern hemisphere $0 \leq \theta \leq \pi / 2+\epsilon$, with the gauge potential (1) and the lower hemishpere $\pi / 2-\epsilon \leq \theta \leq \pi$, with the gauge-transformed potential

$$
\begin{array}{r}
\mathbf{A}_{l}=f_{l}(r, \theta) \hat{\varphi}, \\
f_{l}(r, \theta)=-\frac{g}{4 \pi r} \frac{1+\cos (\theta)}{\sin (\theta)},
\end{array}
$$

corresponding to the same magnetic monopole at the origin but with the Dirac string now along the positive $z$-axis

$$
\mathbf{A}_{l}=\mathbf{A}_{u}-\nabla\left(\frac{g}{2 \pi} \varphi\right)
$$


In both hemispheres the gauge potential is now regular and the corresponding Pauli equation can be solved. The price to pay is gauge transformation between wave functions in the overlap region $[\pi / 2-\epsilon, \pi / 2+\epsilon]$.

The Hamiltonian for two electrons with charges $e$ and masses $m$ and a fixed magnetic monopole with the magnetic charge $g$ at the origin is

$$
\begin{array}{r}
H=\frac{1}{2 m}\left(\mathbf{p}_{1}-e \mathbf{A}\left(\mathbf{x}_{1}\right)\right)^{2}+\frac{1}{2 m}\left(\mathbf{p}_{2}-e \mathbf{A}\left(\mathbf{x}_{2}\right)\right)^{2}-\frac{e}{m} \mathbf{s}_{1} \cdot \mathbf{B}\left(\mathbf{x}_{1}\right) \\
-\frac{e}{m} \mathbf{S}_{2} \cdot \mathbf{B}\left(\mathbf{x}_{2}\right)+V_{\mathrm{R}}\left(\left|\mathbf{x}_{1}-\mathbf{x}_{2}\right|\right)+V_{\mathrm{C}}\left(\left|\mathbf{x}_{1}-\mathbf{x}_{2}\right|\right),
\end{array}
$$

where $\mathbf{A}$ is the monopole gauge potential defined by Eqs. (1) and (2), $\mathbf{s}_{1,2}$ denote the spin vectors of two electrons, $V_{\mathrm{C}}(r)=e^{2} / 4 \pi \varepsilon r$ is the repulsive Coulomb potential, with $\varepsilon$ the relative dielectric permittivity of the material and $V_{\mathrm{R}}(r)$ is the short-range repulsion. We introduce the center of mass and relative coordinates as $\mathbf{R}=\left(\mathbf{x}_{1}+\mathbf{x}_{2}\right) / 2$ and $\mathbf{r}=\left(\mathbf{x}_{1}-\mathbf{x}_{2}\right)$ and we set $\mathbf{R}=0$. To make the model amenable to an analytical solution we make the further simplifying assumption that the infinitely heavy, external magnetic monopole sits exactly at the center of mass of the twoelectron system. The time-independent Pauli equation becomes then

$$
\begin{array}{r}
{\left[-\frac{1}{2 m}\left(\nabla-i e \mathbf{A}\left(\frac{\mathbf{r}}{2}\right)\right)^{2}-\frac{1}{2 m}\left(\nabla+i e \mathbf{A}\left(\frac{-\mathbf{r}}{2}\right)\right)^{2}-\frac{|e g|}{2 \pi m|\mathbf{r}|^{2}}\right.} \\
\left.+V_{\mathrm{R}}(|\mathbf{r}|)+V_{\mathrm{C}}(|\mathbf{r}|)\right] \psi=E \psi,
\end{array}
$$

where we have specialized to a total spin 0 state in which the spin of each electron has a hedgehog configuration parallel or antiparallel to the monopole magnetic field, depending on the sign of $g$.

Suppose now that the motion of electrons is constrained to the two conducting planes at $z= \pm s$, with orbital angular momentum $\pm \ell$ on the upper and lower planes, respectively. If the monopole charge in between satisfies $e g / 2 \pi=-2 \ell$, Eq. (5) reduces to a single $2 \mathrm{D}$ radial equation (see Methods)

$$
\left[-\frac{1}{m}\left(\frac{1}{x} \frac{\partial}{\partial_{x}}\left(x \frac{\partial}{\partial x}\right)\right)+V_{\mathrm{R}}(x)-\frac{|e g / 2 \pi|}{m\left(s^{2}+x^{2}\right)}+V_{\mathrm{C}}(x)\right] F(x)=E F(x),
$$

where $x=r \sin (\theta)$ is the radial distance on the planes and $V_{\mathrm{C}}(x)=$ $\alpha / \varepsilon \sqrt{s^{2}+x^{2}}$. The short-range repulsion models the quantum statistical pressure of electrons when they are squeezed by the two conducting planes. Its exact form does not matter; however, it forces electrons to fall into non-zero orbital angular momentum states $\ell>0$ in order to avoid the energy price to be too close: $F(x) \propto x^{\ell}$ for $x \ll s$ and the higher $\ell$, the more the wave function is suppressed at the origin. Yet, for $e g / 2 \pi=-2 \ell$, the resulting repulsive centrifugal barrier gets completely canceled by the additional, monopole-induced angular momentum, and only an attractive interaction due to the electron magnetic moments survives. Note that these are exactly the values of the monopole charge that leave the individual electron statistics unchanged. In this case, the potential well forms between the two relevant scales $s$ and $a=\varepsilon / m \alpha$, where the Coulomb repulsion takes over, and the electrons form pairs that can Bose condense in a droplet localized around the positions of the heavy monopole. To estimate the optimal value of $\ell$, note that, on one side, increasing $\ell$ makes the magnetic moment attraction stronger, and on the other side, the higher the monopole charge, the heavier they are and the higher the energy cost of creating them between the planes. Given the large mass of monopoles, one expects that at small monopole densities larger values of $\ell$ are favored and vice versa.

The construction of the potential well result again from the interplay of dimensionality. The angular momentum of the electrons constrained to two planes is a 2D effect. The additional angular momentum due to the monopole and the magnetic moment interactions, however, are the $3 \mathrm{D}$ effects, since they are directed from the monopole at the centre to the locations of the electrons on the planes. At sufficiently large distances, the additional angular momentum cancels out the centrifugal barrier and the magnetic moment interaction causes the overall attraction.
The potential well is determined by the combination of the magnetic moment attraction with the short-range repulsion representing quantum statistical pressure of electrons of inter-layer atoms squeezed between the two conducting planes. As such, it should be a scale-free, i.e., $1 / x$ potential. Therefore, in general, both the position of the minimum of the potential well and the bound state energy, are functions of the two spatial scales $s$ and $a$. If they are comparable, there remains only a single spatial and, accordingly, a single energy scale. To estimate it, one can neglect $V_{\mathrm{R}}(x)$ and $V_{\mathrm{C}}(x)$ in eq. (6). By multiplying the whole equation by $m$ we see that the scale $m E$ in the right-hand side is determined by the unique remaining scale $s$ in the left hand-side. Therefore $E_{0}=O\left(1 / m s^{2}\right)$. As shown in ${ }^{39}$, the numerical coefficient is of order one so that

$$
E_{0} \simeq \frac{\hbar^{2}}{m s^{2}}
$$

where we restore physical units. Since the interplane spacing $s \simeq k_{\mathrm{F}}$, with $k_{\mathrm{F}}$ being the Fermi wavevector, $E_{0}=O\left(E_{\mathrm{F}}\right)$. The corresponding localization size of the bound state within the plane is $\ell_{\|} \simeq s$, and as discussed above is the same orthogonal to the plane, $\ell_{\perp} \simeq s$. At finite monopole density $\rho$, when monopoles are brought near each other, the binding energy $E_{0}$ first keeps increasing as long as the two-dimensional parallel to the plane inter-monopole distance $d=(\rho s)^{-1 / 2}>\ell_{\|} \approx s^{40}$. This is the effect of the 2D Lifshitz localization ${ }^{41}$. However, at $d<s$, the "flat" bottoms of the potential wells in the many-monopole generalization of Eq. (6) overlap. Then the pairs are localized by fluctuations in the monopole density rather than by single potential wells, see ${ }^{41}$, where a similar problem was discussed, and $E_{0}$ starts to decrease with increasing $\rho$. Therefore, one expects that the optimal binding is achieved at $d \simeq s$. This prediction for the optimal monopole density is in accord with the recent experimental data of ${ }^{42}$ showing that the highest transition temperature in carbonaceous sulfur is indeed achieved at some optimal distance $s$ between the conducting planes. Exact calculating of $E_{0}$ requires microscopic treatment, but for present purposes the estimate (7) is sufficient.

\section{Discussion and conclusion}

The localized pairs with the binding energy $E_{0}$ are the nucleation centers for superconducting droplets. As we have derived above, the $T=0$ dimension of such droplets is $s$. At higher temperature the droplet dimension $\xi(T)$ will typically be larger. Global superconductivity sets in at the temperature when sufficient monopoles have formed so that these droplets form an infinite three-dimensional cluster and is given by the Ioffe-Larkin ${ }^{43}$ formula for the transition temperature $T_{\mathrm{c}}$ in highly inhomogeneous superconductors

$$
k_{\mathrm{B}} T_{\mathrm{c}}=\omega \mathrm{e}^{-0.89 d / \xi\left(T_{\mathrm{c}}\right)},
$$

where $\xi(T)$ is the characteristic size of a localized pair and $\omega$ is the attempt frequency in the matrix element $t=\omega \exp (-d / \xi)$ describing tunneling of a bound pair between adjacent monopoles. The mean intermonopole distance is itself a function of temperature and material characteristics, but for the estimate we take the optimal monopole density providing the strongest binding, i.e. $d \simeq s$. To favor the factors that can lower the expected $T_{\mathrm{c}}$, however, we take the smallest possible size of the pair, i.e. $\xi \simeq s$. The tunneling matrix element between monopoles at the distance $d$ is found following ${ }^{41}$, and gives $\omega=\sqrt{8 / \pi}\left(\hbar^{2} / m s^{2}\right) \sqrt{s / d}$. The resulting estimate for the transition temperature is

$$
T_{\mathrm{c}} \approx 0.65 \cdot\left[\hbar^{2} /\left(m s^{2} k_{\mathrm{B}}\right)\right]
$$

where we have restored physical units. Taking the interplane distance $s$ as $1 \mathrm{~nm}$, one obtains $T_{\mathrm{c}}=O\left(10^{2}\right) K$.

The proposed real-space monopole pairing mechanism reveals the microscopic nature of HTS. It solves the puzzle why the pairing size in HTS does not exceed the inter-pair distance; this follows from the fact that since the optimal $d \simeq s$, then, in general, $d \gtrsim s$. Therefore the distance between paired electrons is larger than the pair size in HTS. 
Our findings pave the way for tailoring superconducting materials with enhanced $T_{\mathrm{c}}$. To that end, one has to maximally increase the electron density harbored within the adjacent conducting planes, while minimizing the inter-plane distance $s$. The elevated density, by increasing the electronic repulsion, promotes the enhanced generation of monopoles, thereby lowering the system's overall energy. Determining the optimal monopole density and finding the corresponding optimal material parameters requires a detailed self-consistent microscopic treatment of the intertwined electron-monopole ensemble and the derivation of the superconducting transition temperature $T_{\mathrm{c}}$. This self-consistent microscopic theory will be the subject of a forthcoming publication.

\section{METHODS}

Derivation of the Pauli equation on the planes Using that the monopole gauge potentials (1) or (2) are divergenceless, we can simplify equation (5) of the main text to

$$
\begin{aligned}
& {\left[-\frac{1}{m} \nabla^{2}+\frac{i e}{2 m}\left(\mathbf{A}\left(\frac{\mathbf{r}}{2}\right) \cdot \nabla-\mathbf{A}\left(\frac{-\mathbf{r}}{2}\right) \cdot \nabla\right)+\frac{e^{2}}{2 m}\left(\mathbf{A}^{2}\left(\frac{\mathbf{r}}{2}\right)+\mathbf{A}^{2}\left(\frac{-\mathbf{r}}{2}\right)\right)\right.} \\
& \left.-\frac{|e g|}{2 \pi m|\mathbf{r}|^{2}}+V_{\mathrm{R}}(|\mathbf{r}|)+V_{\mathrm{C}}(|\mathbf{r}|)\right] \psi=E \psi .
\end{aligned}
$$

Starting from this generic equation, one has to formulate two Pauli equations, one for each hemisphere, as explained in the main text. Let us begin with the upper hemisphere, denoted by the subscript " $u$ ". Since we restrict to values $0 \leq \theta \leq \pi / 2+\epsilon$, the arguments of the second gauge potentials $\mathbf{A}$ in (10) relate to the lower hemisphere, denoted by subscripts " $l$ ". Therefore, we have to use Eq. (1) for the first instance of the gauge potential and Eq. (2) for the second one. This gives

$$
\begin{aligned}
& {\left[-\frac{1}{m} \nabla^{2}+\frac{i e}{2 m}\left(f_{u}\left(\frac{r}{2}, \theta\right)-f_{l}\left(\frac{r}{2}, \pi-\theta\right)\right) \frac{1}{r \sin (\theta)} \frac{\partial}{\partial \varphi}\right.} \\
& +\frac{e^{2}}{2 m}\left(f_{u}^{2}\left(\frac{r}{2}, \theta\right)+f_{l}^{2}\left(\frac{r}{2}, \pi-\theta\right)\right)-\frac{|e g|}{2 \pi m r^{2}} \\
& \left.+V_{\mathrm{R}}(r)+V_{\mathrm{C}}(r)\right] \psi_{u}=E \psi_{u} .
\end{aligned}
$$

Repeating the same reasoning for the lower hemisphere $\pi / 2-\epsilon \leq \theta \leq \pi$, we obtain the second Pauli equation

$$
\begin{aligned}
& {\left[-\frac{1}{m} \nabla^{2}+\frac{i e}{2 m}\left(f_{l}\left(\frac{r}{2}, \theta\right)-f_{u}\left(\frac{r}{2}, \pi-\theta\right)\right) \frac{1}{r \sin (\theta)} \frac{\partial}{\partial \varphi}\right.} \\
& +\frac{e^{2}}{2 m}\left(f_{l}^{2}\left(\frac{r}{2}, \theta\right)+f_{u}^{2}\left(\frac{r}{2}, \pi-\theta\right)\right)-\frac{|e g|}{2 \pi m r^{2}} \\
& \left.+V_{\mathrm{R}}(r)+V_{\mathrm{C}}(r)\right] \psi_{l}=E \psi_{l} .
\end{aligned}
$$

Using (1) and (2), we obtain, finally, the explicit expressions of our pair of Pauli equations

$$
\begin{array}{r}
{\left[-\frac{1}{m} \nabla^{2}+i \frac{(e g / 2 \pi)}{m r^{2}} \frac{1-\cos (\theta)}{\sin ^{2}(\theta)} \frac{\partial}{\partial \varphi}+\frac{(e g / 2 \pi)^{2}}{m r^{2}}\left(\frac{1-\cos (\theta)}{\sin (\theta)}\right)^{2}\right.} \\
\left.-\frac{|e g|}{2 \pi m r^{2}}+V_{\mathrm{R}}(r)+V_{\mathrm{C}}(r)\right] \psi_{u}=E \psi_{u}, \\
{\left[-\frac{1}{m} \nabla^{2}-i \frac{(e g / 2 \pi)}{m r^{2}} \frac{1+\cos (\theta)}{\sin ^{2}(\theta)} \frac{\partial}{\partial \varphi}+\frac{(e g / 2 \pi)^{2}}{m r^{2}}\left(\frac{1+\cos (\theta)}{\sin (\theta)}\right)^{2}\right.} \\
\left.-\frac{|e g|}{2 \pi m r^{2}}+V_{\mathrm{R}} R(r)+V_{\mathrm{C}}(r)\right] \psi_{l}=E \psi_{l} .
\end{array}
$$

The presence of the magnetic monopole is reflected in three new terms, the first two of which, as anticipated, break the spherical symmetry of the original Coulomb problem. The first embodies the monopole-induced additional contribution to the z-axis component of the angular momentum: it has a different sign in the upper and lower hemispheres since, as we discussed above, it points from the monopole to the electrons and the monopole sits exactly in the middle. Its coefficient is only weakly dependent on $\theta$ since it varies from 1 on the equator to $1 / 2$ at the poles. The second new term, instead is a repulsive term concentrated around the equator and vanishing near the poles. Finally, the third new term is the magnetic attraction due to electron magnetic moments.
Since the vector gauge potentials in the lower and upper hemispheres are gauge transforms of each other, we must impose the Wu-Yang gauge conditions also on the wave functions in the overlap region $[\pi / 2-\epsilon, \pi / 2+\epsilon]$ of the maps of the atlas ${ }^{38}$,

$$
\psi_{l}=\mathrm{e}^{-i \frac{e q}{2 \pi} \varphi} \psi_{u} .
$$

We can thus make the Ansatz

$$
\begin{gathered}
\psi_{u}(r, \theta, \varphi)=\mathrm{e}^{+i \frac{e q}{4 \pi} \varphi} F_{u}(r, \theta, \varphi), \\
\psi_{l}(r, \theta, \varphi)=\mathrm{e}^{-i \frac{e q}{4 \pi} \varphi} F_{l}(r, \theta, \varphi) .
\end{gathered}
$$

Because an exchange of the two electrons involves necessarily also a swap of hemispheres, the exchange operator on the wave function must take into account the Wu-Yang gauge transformation ${ }^{38}$. Therefore, for $e g / 2 \pi$ an odd integer the exchange implies a factor (-1) and the statistics of the electrons is changed to bosons. For $e g / 2 \pi$ an even integer, instead there is no additional $(-1)$ factor and the statistics of the individual electrons remains fermionic. Correspondingly, for $e g / 2 \pi$ an odd integer the additional gauge factor is a double covering representation of $2 \pi$ rotations, while it is single-valued for $e g / 2 \pi$ an even integer. This is the statistical transmutation induced by magnetic monopoles (for a review see ${ }^{31}$ ). Independently of this statistical transmutation of the individual components, however, the total spin 0 pair is a boson.

We now constrain the electron motion to two parallel horizontal planes at $z= \pm s$ with the monopole at the origin. As a consequence, the Laplace operator reduces to

$$
\nabla^{2}=\frac{\partial^{2}}{\partial x^{2}}+\frac{1}{x} \frac{\partial}{\partial x}+\frac{1}{x^{2}} \frac{\partial^{2}}{\partial \varphi^{2}},
$$

where $x$ denotes the radial distance on the two planes. We are interested primarily in small values of $x$ and the second new term in (13), $O\left(\theta^{2}\right)$, is subdominant with respect to the first one, $O(1)$ near the poles: we will henceforth neglect it. In addition, since the electrons are forced to move on the two horizontal planes, we do not have to use the usual monopole harmonics ${ }^{38}$ but we can make use of the much simpler cylindrical harmonics decomposition by making the Anstaz

$$
\begin{aligned}
F_{u}(r, \theta, \varphi) & =\mathrm{e}^{+i \ell \varphi} F(x), \\
F_{l}(r, \theta, \varphi) & =\mathrm{e}^{-i \ell \varphi} F(x),
\end{aligned}
$$

where $r$ and $\theta$ are bound by the condition $x=r \sin \theta$ and $\ell$ is the angular momentum. The $z$-components of the angular momentum of the electrons on the two planes cancel out, but the total angular momentum $|\ell|$ can well be different from zero. It is this value that indicates how much the axis of the composite wave function is tilted with respect to the $z$-axis. Combining (17) with (15) gives the announced result. When $e g / 2 \pi=-2 \ell$ the total angular momentum and the ensuing centrifugal barrier vanish altogether. We obtain thus a single radial equation for both planes, Eq. (6) of the main text.

\section{Data availability}

Data sharing not applicable to this article as no datasets were generated or analyzed during the current study.

\section{References}

1. Barišić, N. et al. Universal sheet resistance and revised phase diagram of the cuprate high-temperature superconductors. PNAS 110, 12235 - 12240 (2013).

2. Keimer, B., Kivelson, S. A., Norman, M. R., Uchida, S. \& Zaanen, J. From quantum matter to high-temperature superconductivity in copper oxides. Nature $\mathbf{5 1 8}$, 179-186 (2015).

3. Božović, I. \& Levy, J. Pre-formed Cooper pairs in copper oxides and $\mathrm{LaAlO}_{3}$ $\mathrm{SrTiO}_{3} 3$ heterostructures. Nature Physics 16, 712 - 717 (2020).

4. Diamantini, M. C., Trugenberger, C. A. \& Vinokur, V. M. Topological Nature of High Temperature Superconductivity. Advanced Quantum Technologies 2021, 2000135 (2020).

5. Bussman-Holder, A. \& Keller, H. High-temperature superconductors: underlying physics and applications. Z. Naturforsch. 75 (1-2)b, 3-14 (2020)

6. Anderson, P. W. The Resonating Valence Bond State in $\mathrm{La} 2 \mathrm{CuO} 4$ and Superconductivity. Science 235, 1196 - 1198 (1987).

7. Harland, M., Katsnelson, M. I. \& Lichtenstein, A. I. Plaquette valence bond theory of high-temperature superconductivity. Phys. Rev. B 94, 125133 (2016). 
8. Monthoux, P., Balatsky, A. V. \& Pines, D. Toward a theory of high-temperature superconductivity in the antiferromagnetically correlated cuprate oxides. Phys. Rev. Lett. 67, 3448 - 3451 (1991).

9. Chubukov, A. V. \& Schmalian, J. Superconductivity due to massless boson exchange in the strong-coupling limit. Phys. Rev. B 72, 174520 (2005).

10. Sato, Y. et al. Thermodynamic evidence for a nematic phase transition at the onset of the pseudogap in $\mathrm{YBa}_{2} \mathrm{Cu}_{3} \mathrm{O}_{\mathrm{y}}$. Nature Physics 13, $1074-1078$ (2017).

11. Zhou, P. et al. Electron pairing in the pseudogap state revealed by shot noise in copper oxide junctions. Nature 572, 493 (2019).

12. Proust, C. \& Taillefer, L. The Remarkable Underlying Ground States of Cuprate Superconductors. Annu. Rev. Condens. Matter Phys. 10, 409 - 429 (2019).

13. Mukhopadhyay, S. et al., Evidence for a vestigial nematic state in the cuprate pseudogap phase. Proc. Natl. Acad. Sci. U.S.A. 116, 13249 - 13254 (2019).

14. Kivelson, S. A., Fradkin, E. \& Emery, V. J. Electronic liquid-crystal phases of a doped Mott insulator. Nature, 393, 550 - 553 (1998).

15. Zheng, G. Q., Kuhns, P. L., Reyes, A. P., Liang, B. \& Lin, C. T. Critical point and the nature of the pseudogap of single-layered copper-oxide $\mathrm{Bi}_{2} \mathrm{Sr}_{2-x} \mathrm{La}_{x} \mathrm{CuO}_{6+\delta}$ superconductors. Phys. Rev. Lett. 94, 047006 (2005).

16. Nie, L., Tarjus, \& Kivelson, S. A. Quenched disorder and vestigial nematicity in the pseudogap regime of the cuprates. PNAS, 111, 7980 - 7985 (2014).

17. Chubukov, A. \& Hirschfeld, P. J. Iron-based superconductors, seven years later. Physics Today 68, 46 - 52 (2015).

18. Achkar, A. J. et al. Nematicity in stripe-ordered cuprates probed via resonant X-ray scattering. Science 351, $576-578$ (2016).

19. Kivelson, S. A. \& Lederer, S. Linking the pseudogap in the cuprates with local symmetry breaking: A commentary. PNAS 116, 14395 - 14397 (2019).

20. de Carvalho, V.S., Chubukov, A. V. \& Fernandes, R. M. Thermodynamic signatures of an antiferromagnetic quantum critical point inside a superconducting dome. Phys. Rev. B 102, 045125 (2020).

21. Chichinadze, D. V., Classen, L. \& Chubukov, A. V. Nematic superconductivity in twisted bilayer graphene. arXiv:1910.07379v2 (2020).

22. Chubukov, A. V. \& Schmalian, J. The pairing glue in cuprate superconductors from the self-energy revealed via machine learning. arXiv:2002.02994v1 (2020).

23. Sacépé, B., Feigel'man, M. \& Klapwijk, T. M. Quantum breakdown of superconductivity in low-dimensional materials. Nature Physics, 16, $734-746$ (2020).

24. Diamantini M. C., Sodano, P. \& Trugenberger, C. A. Gauge theories of Josephson junction arrays. Nuclear Physics B 474, 641 - 677 (1996).

25. Vinokur, V. M. et al. Superinsulator and quantum synchronization. Nature $\mathbf{4 5 2}, 613$ -615 (2008).

26. Diamantini, M. C. et al. Bosonic topological insulator intermediate state in the superconductor-insulator transition. Physics Letters A 384, 126570 (2020).

27. Diamantini, M.C., Trugenberger, C. A. \& Vinokur, V.M. Quantum magnetic monopole condensate. Communications Physics 4, 25 (2021).

28. Diamantini, M. C., Trugenberger, C. A. \& Vinokur, V. M. Confinement and asymptotic freedom with Cooper pairs. Communications Physics 1, 77 (2018).

29. Trugenberger, C., Diamantini, M.C., Poccia, N., Nogueira, F. S., Vinokur, V. M. Magnetic Monopoles and Superinsulation in Josephson Junction Arrays. Quantum Rep. 2, 388-399 (2020).

30. Chubukov, A. V., Abanov, A. Esterlis, I. \& Kivelson, S. A. Eliashberg theory of phonon-mediated superconductivity - when it is valid and how it breaks down. arXiv:2004.01281v1 [cond-mat.supr-con] (2020).
31. Goddard, P. and Olive, D. I. Magnetic monopoles in gauge fields theories, Rep. Progr. Phys. 411357 - 1437 (1978).

32. Preskill, J. Magnetic monopoles. Ann. Rev. Nucl. Part. Sci. 34 461-530 (1984).

33. Pomar, A., Ramallo, M. V., Mosqueira, J., Torrón, C. \& Vidal, F. Fluctuationinduced in-plane conductivity, magnetoconductivity, and diamagnetism of $\mathrm{Bi}_{2} \mathrm{Sr}_{2} \mathrm{CaCu}_{2} \mathrm{O}_{8}$ single crystals in weak magnetic fields. Phys. Rev. B 54, 7470 - 7480 (1996).

34. Zhao, F. et al. Sign-Reversing Hall Effect in Atomically Thin High-Temperature $\mathrm{Bi}_{2.1} \mathrm{Sr}_{1.9} \mathrm{CaCu}_{2.0} \mathrm{O}_{8+\delta}$ Superconductors. Phys. Rev. Lett. 122, 247001 (2019).

35. Kapitulnik, A., Xia, J., Schemm, E., Palevski, A. Polar Kerr effect as probe for time- reversal symmetry breaking in unconventional superconductors. New J. Phys. 11, 055060 (2009).

36. Yu, Y. et al. High-temperatures superconductivity in monolayer $\mathrm{Bi}_{2} \mathrm{Sr}_{2} \mathrm{CaCu}_{2} \mathrm{O}_{8+\delta}$. Nature 575, 156 - 163 (2019).

37. Dorin, V. V., Klemm, R. A., Varlamov, A. A., Buzdin, A. I. \& Livanov, D. V. Fluctuation conductivity of layered superconductors in a perpendicular magnetic field. Phys. Rev. B 48, 12951 - 12965 (1993).

38. Wu, T. T. \& Yang, C. N. Dirac monopole without strings: monopole harmonics. Nucl. Phys. B 107, 365-380 (1976).

39. Blatter, G., Feigel'man M. V., Geshkenbein, V. B., Larkin, A. I. \& Vinokur, V. M. Vortices in high-temperature superconductors. Rev. Mod. Phys. 66, 1125 - 1388 (1994).

40. Baz, A. I., Perelomov, A. M., Zel'dovich, Y.B. Scattering, reactions and decay in nonrelativistic quantum mechanics. Jerusalem: Israel Program for Scientific Translations, 1969

41. Nelson, D. R. \& Vinokur, V. M. Boson localization and correlated pinning of superconducting vortex arrays. Phys. Rev. B 48, 13060 - 13097 (1993).

42. Snider, E. et al., Room-temperature superconductivity in a carbonaceous sulfur hydride. Nature 586, 373-377 (2020).

43. Ioffe, L. B. \& Larkin, A. I. Properties of superconductors with a smeared transition temperature. Sov. Phys. JETP 54, 378 - 384 (1981).

Acknowledgements We are delighted to thank Andrey Chubukov and Alexander Golubov for illuminating discussions. The work by V.M.V. was supported by Terra Quantum AG. M.C.D. thanks CERN, where she completed this work, for kind hospitality.

Author contribution M.C.D., C.A.T., and V.M.V conceived the work, carried out the calculations, and wrote the manuscript.

Competing interests The Authors declare that they have no competing interests.

Correspondence Correspondence and requests for materials should be addressed to V.M.V. (vmvinokour@gmail.com). 